\title{
A stage-structured delayed advection reaction-diffusion model for single species
}

\author{
Raed Ali Alkhasawneh \\ College of Applied Studies and Community Service, Imam Abdulrahman Bin Faisal University, Saudi Arabia
}

\begin{tabular}{|c|c|}
\hline Article Info & ABSTRACT \\
\hline Article history: & In this paper, we derived a delay advection reaction-diffusion equation with \\
\hline Received Mar 13, 2020 & $\begin{array}{l}\text { linear advection term from a stage-structured model, then the derived } \\
\text { equation is used under the homogeneous Dirichlet boundary conditions }\end{array}$ \\
\hline Revised Jun 6, 2020 & $u_{m}(0, t)=0, u_{m}(L, t)=0$, and the initial condition $u_{m}(x, 0)=u_{m}^{0}(x)$ \\
\hline Accepted Jun 16, 2020 & $\begin{array}{l}>0, x \in[-\tau, 0] \text { with } u_{m}^{0}(0)>0 \text { in order to find the minimum value of } \\
\text { domain } L \text { that prevents extinction of the species under the effect of advection }\end{array}$ \\
\hline Keywords: & $\begin{array}{l}\text { reaction-diffusion equation. Finally, for the measurement the time lengths } \\
\text { from birth to the development of the species population, time delays }\end{array}$ \\
\hline Competition & are \\
\hline
\end{tabular}

Stage structure

Time delay

Advection

Travelling wave solutions

Copyright (C) 2020 Institute of Advanced Engineering and Science. All rights reserved.

\section{Corresponding Author:}

Raed Ali Alkhasawneh,

Department of General Courses,

Imam Abdulrahman Bin Faisal University,

P. O. Box 1982, Dammam, 31441, Saudi Arabia.

Email: ralkhasawneh@iau.edu.sa

\section{INTRODUCTION}

Reaction-diffusion conditions demonstrate are exceptionally critical in numerous individuals' developments in an environment or media and nearby response energy such as birth, passing and other response handle, see Britton [1], Lewis [2], Allen [3] and Aiello [4]. Besides, reaction-diffusion frameworks can be clarified the impacts of the measure, shape and heterogeneity of the spatial environment on the diligence of species and the structure of communities in environment. Reaction $(f(u))$ and dispersal (spread coefficient $D$ ) both contribute to the inquisitively dynamical behavior of the arrangements of the condition. We are able to describe the over component by the classical FKPP (Fisher's condition) reaction-diffusion condition $[5,6]$ :

$$
\frac{\partial u}{\partial t}=f(u)+D \frac{\partial^{2} u}{\partial x^{2}}
$$

where $f(u)=u(1-u)$.

It can consider the diffusion as an irregular step which begins at a point and takes place in an arbitrary heading. Fick's laws can be utilized to solve for the diffusion coefficient $D$ and it addresses that the diffusive flux goes from areas of high concentration to areas of low concentration. The extraordinary arrangement of the (1) could be an engendering front (moreover called traveling wave arrangement), the two non-equilibrium homogeneous states are isolated, one of which $(u=1)$ is steady and another one $(u=0)$ is unsteady [7-9]. For illustration, Gurney and Nisbet [8] expanded the collection of stage-structure models which can be portrayed in terms of delay-differential conditions by analyzing models where the processes of development and improvement within a stage are distinct. This grants the utilize of delay-differential 
condition models in circumstances where both populace numbers and add up to biomass are powerfully critical. Al-Omari and Gourley $[10,11]$ proposed a delay differential condition show for a single species with stage-structure in which the development delay is displayed as a dissemination, to permit for the possibility that people may take diverse sums of time to develop. Common birth and passing rate capacities are utilized. They found that the elements of the demonstrate depends to a great extent on the qualitative frame of the birth work, which depends on the overall number of grown-ups. They also propose an alternative model which derived by Aiello, Freedman and $\mathrm{Wu}$, see [12], allowing a non-monotone birth function of the kind seen in other frequently studied population models. Then they present results on positivity and boundedness, existence and local stability of equilibria, global stability of the extinction state under certain conditions, and existence of periodic solutions. Gourley et al., [13] presented demonstrate circuitous transmission, through contact with infections, of avian influenza in transitory and nonmigratory fowls, taking under consideration age structure. Relocation is modeled through a reaction-advection condition on a closed circle parametrized by bend length (the migration flyway) that begins and closes at the area where fowls breed in summer. Their modeling keeps the winged creatures together as a run, the position of which is certainly decided and known for all future time. Births happen when the run passes the breeding area and are modeled utilizing thoughts of rash differential conditions. Sarfaraz [14] classified the parameter spaces for reaction-diffusion frameworks of two chemical species on stationary spaces in terms of the sorts and solidness of the uniform consistent state and irritated the impact on the flow of the re-action-diffusion framework.

Chunwei [15] defined a postponed reaction-diffusion demonstrate that depicts competition between two species in a stream. He isolates each species into two champers, people in-habiting on the seabed and people floating within the stream. Time delays are consolidated to degree the time lengths from birth to development of the benthic populaces. It too ponders the elements of the non-spatial show, decide the presence and worldwide solidness of the equilibrium, and give conditions beneath which arrangements merge to the balance. At that point he appears that the presence of traveling wave arrangements can be set up through compact fundamentally operators. At last, he defines two genuine numbers and demonstrate that they serve as the lower bounds of the speeds of traveling wave arrangements within the framework.

Rattanakul [16] built an advection diffusion-reaction model demonstrate for the angle populace structured to track the populace densities of both the labeled angle and the tag-free angle, in which the rash labeling hone is joined, whereas nonstop labeling is accepted to be done on off springs of tractable labeled angle, or on those within the same swarm as the tractable labeled angle. Utilizing the traveling wave facilitate, they infer explanatory expression for the arrangements to the show framework. Also, they inferred the unequivocal expression for the level of labeled angle which increments in an intermittent rash design. Solidness and stage plane investigations are moreover carried out to determine. While Venkataraman [17] investigate a model for biological pattern formation during growth development. The pattern formation phenomenon is described by a reaction-diffusion system on a time-dependent domain.

Boonrangsiman et al. [18] considered an application to fisheries and accepted that there's a single prey populace and a predator populace that can be isolated by generation capacity into a youthful and a develop arrange, with a time delay for the youthful to develop move. They demonstrated that the framework has three nonnegative balance focuses, specifically, an unimportant point with all populaces zero, a predatorfree harmony point, and a coexistence balance point with all three populaces non-zero. It is demonstrated that the minor harmony point is continuously unsteady, that the predator-free balance point is steady on the off chance that and as it were in the event that the existence harmony point does not exist, which the existence point can either be steady for all time delays or ended up unsteady in the event that a Hopf bifurcation occurs at a basic time delay. While Zhang [19] explored a stage-structured postponed reaction-diffusion show with advection that depicts competition between two develop species in water stream. Time delays are consolidated to degree the time lengths from birth to development of the populaces. They appear that there exists a limited positive number $\mathrm{c}^{*}$ that can be characterized as the slowest spreading speed of traveling wave arrangements interfacing two mono-culture equilibria or interfacing a monoculture with the coexistence harmony. Moreover, Cangiani et al. [20] illustrated a few modern sorts of wave proliferation and design arrangement in a classical three species cyclic competition show with spatial dissemination These unused designs are characterized by a tall normality in space but are diverse from designs already known to exist in reaction-diffusion models and demonstrated a dependable bound for the blunder of the numerical strategy which permit us to effectively investigate the dynamical designs in both two and three spatial measurements. At last, Murray [21-23] examined the condition for the linear stability of $u=0$ for the taking after response dissemination demonstrate:

$$
\frac{\partial u}{\partial t}=f(u)+D \frac{\partial^{2} u}{\partial x^{2}}, \quad 0 \leq x \leq L
$$


With the following boundary and initial conditions:

$$
u(x, t)=0, u(L, t)=0, u(x, 0)=u_{0}(x),
$$

where $u$ denotes the population density of a species, $f(u)$ is the species dynamics and $D$ is the diffusion coefficient which measure the dispersal efficiency of the relevant species, $L$ is the length of the domain. He considered the different boundary conditions which consider the influence of the region exterior to the reaction diffusion domain. He showed that if the domain size is not large enough, then (2) cannot generate spatial patterns and so he found the condition on $L$ such that the species will not become extinct if:

$$
L>\pi \sqrt{\frac{D}{f^{\prime}(0)}}
$$

In this paper, an age-structured model is used to derive a delay advection reaction-diffusion equation with linear advection term. In addition, we find the minimum value of $L$ that prevents extinction of the species under the effect of advection term for the reaction-diffusion equation.

\section{A DELAY ADVECTION REACTION-DIFFUSION EQUATION WITH LINEAR ADVECTION TERM}

In this section, we derive a delay advection reaction-diffusion equation with linear advection term from an age-structured model, and then later on, we study and analyze our new model under the effect of the advection term. Define $u_{m}(x, t)$ to be the intensity of mature adults, with age of at least $\tau$. Then it can be represented as follows:

$$
u_{m}(x, t)=\int_{\tau}^{\infty} u(x, t, a) d a
$$

where $u(x, t, a)$ is the intensity of individuals of age $a$ at point $x$, time $t$ and $\tau$ is the length of the juvenile period. Now, let $u$ satisfies the following equation, see Metz [24] and McNair [25]:

$$
\frac{\partial u}{\partial t}+\frac{\partial u}{\partial a}=U \frac{\partial u}{\partial x}+d_{i} \frac{\partial^{2} u}{\partial x^{2}}-\gamma u, 0<a<\tau
$$

and that the mature evolve according to:

$$
\frac{\partial u_{m}}{\partial t}=U \frac{\partial u_{m}}{\partial x}+d_{m} \frac{\partial^{2} u_{m}}{\partial x^{2}}+u(x, t, \tau)-\beta u_{m}^{2}, \quad x \in(-\infty, \infty), t>0
$$

with

$$
u(x, t, 0)=f\left(u_{m}(x, t)\right)
$$

the term $u(x, t, \tau)$ in (5) represents adult recruitment, since it is those of age $\tau$ (maturation age) and $f\left(u_{m}\right)$ in (6) refers to the rare of the birth, supposed to depend only on the number of matures at the time.

Let $v(x, r, a)=u(x, a+r, a)$, then

$$
\begin{aligned}
& \frac{\partial v}{\partial a}=\left[\frac{\partial u}{\partial t}(x, t, a)+\frac{\partial u}{\partial a}(x, t, a)\right]_{t=a+r} \\
& =U \frac{\partial u}{\partial x}(x, a+r, a)+d_{i} \frac{\partial^{2} u}{\partial x^{2}}(x, a+r, a)-\gamma u(x, a+r, a),
\end{aligned}
$$

which implies

$$
\frac{\partial v}{\partial a}=U \frac{\partial v}{\partial x}+d_{i} \frac{\partial^{2} v}{\partial x^{2}}-\gamma v \frac{\partial u}{\partial t}+\frac{\partial u}{\partial a}=U \frac{\partial u}{\partial x}+d_{i} \frac{\partial^{2} u}{\partial x^{2}}-\gamma u, 0<a<\tau,
$$


Now, we apply the Fourier transform:

$$
\hat{v}(s, r, a)=\int_{-\infty}^{\infty} v(x, r, a) e^{-i s x} d x
$$

To (8) gives:

$$
\frac{\partial \hat{v}}{\partial a}=\left(U i s-d_{i} s^{2}-\gamma\right) \hat{v}
$$

and

$$
\hat{v}(s, r, 0)=\hat{u}(s, r, 0)=\mathcal{F}\left\{f\left(u_{m}(x, r)\right)\right\}
$$

The solution of (9) is:

$$
\hat{v}(s, r, a)=\mathcal{F}\left\{f\left(u_{m}(x, r)\right)\right\} e^{\left(\text {Uis }-d_{i} s^{2}-\gamma\right) a} .
$$

Now,

$$
\begin{aligned}
& \mathcal{F}^{-1}\left(e^{\left(U i s-d_{i} s^{2}-\gamma\right) a}\right)=\frac{1}{2 \pi} e^{\left(U i s-d_{i} s^{2}-\gamma\right) a} e^{i s x} d s \\
& =\frac{e^{-\gamma a}}{\sqrt{4 \pi d_{i} a}} e^{-(x+U a)^{2} /\left(4 d_{i} a\right)} .
\end{aligned}
$$

By using convolution theorem:

$$
v(x, r, a)=e^{-\gamma a} \int_{-\infty}^{\infty} \frac{1}{\sqrt{4 \pi d_{i} a}} e^{\frac{-(U a+x-y)^{2}}{4 d_{i} a}} f\left(u_{m}(y, r)\right) d y,
$$

Therefore

$$
\begin{gathered}
u(x, t, \tau)=v(x, t-\tau, \tau) \\
=e^{-\gamma \tau} \int_{-\infty}^{\infty} \frac{1}{\sqrt{4 \pi d_{i} \tau}} e^{\frac{-(U \tau+x-y)^{2}}{4 d_{i} \tau}} f\left(u_{m}(y, t-\tau)\right) d y .
\end{gathered}
$$

If $f\left(u_{m}\right)=\alpha u_{m}$, then the birth rate is symmetric to the number of the mature adults given by $\alpha u_{m}$. Most of the populations, such presumption is valid only when population numbers are relatively little or when there is abundance of nourishment. Inserting (12) with $f\left(u_{m}\right)=\alpha u_{m}$ into (4) yields:

$$
\frac{\partial u_{m}}{\partial t}=U \frac{\partial u_{m}}{\partial x}+d_{m} \frac{\partial^{2} u_{m}}{\partial x^{2}}-\beta u_{m}^{2}+\propto e^{-\gamma \tau} \int_{-\infty}^{\infty} \frac{1}{\sqrt{4 \pi d_{i} \tau}} e^{\frac{-(U \tau+x-y)^{2}}{4 d_{i} \tau}} u_{m}(y, t-\tau) d y
$$

By applying the following substitution in (13):

$$
U \tau+x-y=w \sqrt{4 d_{i} \tau}
$$

we obtain:

$$
\begin{aligned}
\alpha e^{-\gamma \tau} \int_{-\infty}^{\infty} \frac{1}{\sqrt{4 \pi d_{i} \tau}} e^{\frac{-(U \tau+x-y)^{2}}{4 d_{i} \tau}} u_{m}(y, t-\tau) d y \\
\quad=\alpha e^{-\gamma \tau} \int_{-\infty}^{\infty} \frac{1}{\sqrt{\pi}} e^{-w^{2}} u_{m}\left(U \tau+x-w \sqrt{4 d_{i} \tau}, t-\tau\right) d w
\end{aligned}
$$

This form of the integral allows us to make approximation for small values of the parameters. Let us approximate (14) for small values of the immature diffusivity $d_{i}$ : 


$$
\begin{aligned}
& u_{m}\left(x+U \tau-w \sqrt{4 d_{i} \tau}, t-\tau\right) \\
& =u_{m}(x+U \tau, t-\tau)-w \sqrt{4 d_{i} \tau} \frac{\partial u_{m}}{\partial x}(x+U \tau, t-\tau)+2 d_{i} \tau w^{2} \frac{\partial^{2} u_{m}}{\partial x^{2}}(x+U \tau, t-\tau)
\end{aligned}
$$

Therefore, the integral term in (14) can be written as follows:

$$
\begin{aligned}
& \alpha e^{-\gamma \tau} \int_{-\infty}^{\infty} \frac{1}{\sqrt{\pi}} e^{-w^{2}}\left(u_{m}\left(x+U \tau-w \sqrt{4 d_{i} \tau}, t-\tau\right)\right) d w \\
& \left.=\alpha e^{-\gamma \tau} u_{m}(U \tau+x, t-\tau)+\alpha e^{-\gamma \tau} d_{i} \tau \frac{\partial^{2} u_{m}}{\partial x^{2}}(x+U \tau, t-\tau)\right),
\end{aligned}
$$

where we have used the following fact:

$$
\int_{-\infty}^{\infty} w^{2} e^{-w^{2}} d w=\frac{1}{2} \sqrt{\pi}
$$

In the limiting case $d_{i} \rightarrow 0$ (i.e., immatures not diffusing at all), (13) becomes:

$$
\frac{\partial u_{m}}{\partial t}=U \frac{\partial u_{m}}{\partial x}+d_{m} \frac{\partial^{2} u_{m}}{\partial x^{2}}+e^{-\gamma \tau} u_{m}(x+U \tau, t-\tau)-\beta u_{m}^{2}
$$

In this case $d_{i}=0$, the term $\alpha e^{-\gamma \tau} u_{m}(x+U \tau, t-\tau)$ can be understood ecologically as follows: Setting $d_{i}=0$ means the immature are not diffusing. They are, however, still subject to advection. During the period of immaturity, which is of duration $\tau$, each immature will have moved a distance equal to $U \tau$. It follows that an individual that becomes mature at location $\mathrm{x}$ must have been born at location $x+U \tau$ (since the advection is from right to left). The term: $\alpha e^{-\gamma \tau} u_{m}(x+U \tau, t-\tau)$, which is the rate at time $t$, position $x$, at which individuals become mature, is given simply by the birth rate $\tau$ time units ago at location $x+U \tau$, $\alpha u_{m}(x+U \tau, t-\tau)$, corrected for juvenile mortality which explains the $e^{-\gamma \tau}$ factor. If the species is close to extinction and the immature are not diffusing, then (16) becomes:

$$
\frac{\partial u_{m}}{\partial t}=U \frac{\partial u_{m}}{\partial x}+d_{m} \frac{\partial^{2} u_{m}}{\partial x^{2}}+\alpha e^{-\gamma \tau} u_{m}(x+U \tau, t-\tau), \quad 0<x<L, \quad t>0
$$

\section{EFFECT OF ADVECTION TERM ON THE DERIVED DELAY ADVECTION REACTION- DIFFUSION EQUATION}

Starting by studying (17) on homogeneous Dirichlet boundary conditions: $u_{m}(0, t)=0$, $u_{m}(L, t)=0$, and the initial condition $u_{m}(x, 0)=u_{m}^{0}(x), x \in[-\tau, 0]$ with $u_{m}^{0}(0)>0$. As a starting point, we shall also consider the case when the juveniles are not subject to advection, in this case, the term $u_{m}(x+$ $U \tau, t-\tau)$ of (16) becomes: $\alpha e^{-\gamma \tau} u_{m}(x, t-\tau)$. However, the adults will still be subject to advection. Seeking trial solutions for (16) of the form $u_{m}(x, t)=e^{\sigma \tau} \phi(x)$, yields:

$$
\left.d_{m} \phi^{\prime \prime}(x)+U \phi^{\prime-\gamma \tau} e^{-\sigma \tau}-\sigma\right) \phi(x)=0
$$

The characteristic equation for (18) is

$$
d_{m} M^{2}+U M+\left(\alpha e^{-\gamma \tau} e^{-\sigma \tau}-\sigma\right)=0
$$

and the roots are given by:

$$
M_{1}, M_{2}=\frac{1}{2 d_{m}}\left(-U \pm\left(\sqrt{U^{2}-4 d_{m}\left(\alpha e^{-\gamma \tau} e^{-\sigma \tau}-\sigma\right)}\right)\right.
$$

Thus, we have two cases for the roots:

If $M_{1}$ and $M_{2}$ are real, then the solution for $\phi$ satisfying the boundary condition $\phi(0)=0$ will be in the form

$$
\phi(x)=A\left(e^{M_{1} x}-e^{M_{2} x}\right)
$$


and in order to satisfy $\phi(L)=0$, we will have the zero solution.

If $\mathrm{M}_{1}$ and $\mathrm{M}_{2}$ are complex, then the solution for $\phi(x)$ satisfying the boundary condition $\phi(0)=0$ will be of the form

$$
\phi(x)=e^{\left(\frac{-U x}{2 d_{m}}\right)} B \sin \left(\sqrt{\frac{4 d_{m}\left(\alpha e^{-\gamma \tau} e^{-\sigma \tau}-\sigma\right)-U^{2}}{2 d_{m}}} x\right) .
$$

In order to be able to satisfy $\phi(L)=0$, we need:

$$
\frac{\sqrt{\left(4 d_{m}\left(\alpha e^{-\gamma \tau} e^{-\sigma \tau}-\sigma\right)-U^{2}\right)}}{2 d_{m}}=\frac{n \pi}{L}, \quad n=1,2,3, \ldots
$$

That is:

$$
4 d_{m}\left(\alpha e^{-\gamma \tau} e^{-\sigma \tau}-\sigma\right)-U^{2}=\frac{4 d_{m}^{2} n^{2} \pi^{2}}{L^{2}}, n=1,2,3, \ldots
$$

which gives the following equation to be solved for $\sigma$ :

$$
\sigma=\alpha e^{-\gamma \tau} e^{-\sigma \tau}-\frac{U^{2}}{4 d_{m}}-\frac{d_{m} n^{2} \pi^{2}}{L^{2}}
$$

The solution $u_{m}(x, t)$ of the linearized (17) is of the following form

$$
u_{m}(x, t)=e^{\sigma t} B_{n} e^{\frac{-U x}{2 d_{m}}} \sin \left(\frac{n \pi x}{L}\right), \quad n=1,2,3, \ldots
$$

where $\sigma$ satisfies (20). to obtain:

Now, we are going to find the solution of (17) satisfying the initial data by summing over $n$

$$
u_{m}(x, t)=\sum_{n=1}^{\infty} B_{n} e^{\sigma_{n} t} e^{\frac{-U x}{2 d_{m}}} \sin \left(\frac{n \pi x}{L}\right)
$$

where $\sigma_{n}$ satisfies (21) and using the initial condition and Fourier coefficients, then the value of $B_{n}$ is given by:

$$
B_{\{n\}}=\frac{2}{L} \int_{0}^{L} u_{m}^{0}(x) e^{\frac{U x}{2 d_{m}}} \sin \left(\frac{n \pi x}{L}\right) d x, \quad n=1,2,3, \ldots
$$

Substituting this value in (22) yields the final solution of the linearized problem:

$$
u_{m}(x, t)=\sum_{n=1}^{\infty}\left(\frac{2}{L} \int_{0}^{L} u_{m}^{0}(x) e^{\frac{U x}{2 d_{m}}} \sin \left(\frac{n \pi x}{L}\right) d x \times e^{\sigma_{n} t} e^{\frac{-U x}{2 d_{m}}} \sin \left(\frac{n \pi x}{L}\right)\right.
$$

The population will decay to zero if $\operatorname{Re} \sigma_{n}<0$, for all $n=1,2,3, \ldots$, where $\sigma_{n}$ is any root of (21). In other words, $\sigma_{n}$ satisfies:

$$
\sigma_{n}=\alpha e^{-\gamma \tau} e^{-\sigma_{n} t}-\frac{d_{m} n^{2} \pi^{2}}{L^{2}}-\frac{U^{2}}{4 d_{m}}
$$

In order to detect a possible change of linear stability of the zero solution of (17) we set $\sigma=i \omega$, which gives:

$$
\frac{U^{2}}{4 d_{m}}+\frac{d_{m} n^{2} \pi^{2}}{L^{2}}+i w=\alpha e^{-\gamma \tau} e^{-i w t}
$$


and the complex conjugate of above equation is:

$$
\frac{U^{2}}{4 d_{m}}+\frac{d_{m} n^{2} \pi^{2}}{L^{2}}-i w=\alpha e^{-\gamma \tau} e^{i w t},
$$

Thus, we are eliminating $e^{-i \omega \tau}$ from the last two equations, yields:

$$
\omega^{2}=\alpha^{2} e^{-2 \gamma \tau}-\left[\frac{U^{2}}{4 d_{m}}+\frac{d_{m} n^{2} \pi^{2}}{L^{2}}\right]^{2} .
$$

To find the conditions on the parameters which ensure that: $\omega^{2}<0$, since this means that roots of (21) of the form $\sigma=i \omega$, with $\omega$ real, cannot exist. Note that: If $\omega^{2}<0$ when $n=1$ then automatically $\omega^{2}<0$ for $\mathrm{n}=2,3,4, \ldots$, therefore it is sufficient to set $n=1$. When $n=1, \omega^{2}<0$ and if the following inequality holds:

$$
\alpha e^{-\gamma \tau}<\frac{U^{2}}{4 d_{m}}+\frac{d_{m} \pi^{2}}{L^{2}}
$$

then $u_{m}(x, t) \rightarrow 0$ as $t \rightarrow 0$, thus the population will become extinct. So, the species will become extinct in this case if:

$U$ (advection speed) is large

$d_{m}$ (mature diffusion) is either very small or very large

$L$ (length of domain) is small

$\alpha$ (birth rate) is too small

$\gamma$ (juvenile mortality) is large

$\tau$ (length of juvenile period) is large.

Also, if the following inequality holds:

$$
\alpha e^{-\gamma \tau}>\frac{U^{2}}{4 d_{m}}+\frac{d_{m} \pi^{2}}{L^{2}}
$$

then $\sigma_{n}=\alpha e^{-\gamma \tau} e^{-\sigma_{n} t}-\frac{d_{m} n^{2} \pi^{2}}{L^{2}}-\frac{U^{2}}{4 d_{m}}$ with $n=1$ has a real positive root $\sigma$. This can be shown by drawing the graphs of $\frac{U^{2}}{4 d_{m}}+\frac{d_{m} \pi^{2}}{L^{2}}+\sigma$ and $\alpha e^{-\gamma \tau} e^{-\sigma t}$ against $\sigma$, where we can see that the two functions have a real positive root $\sigma$ which means that the zero solution of (17) is unstable. So, we can conclude that the population will survive in this case if

$U$ (advection speed) is too small.

$L$ (domain) is too large.

$\alpha$ (birth rate) is too large.

\section{DISCUSSIONS AND CONCLUSION}

We have derived a delay advection reaction-diffusion equation with linear advection term from an age-structured model which is given by:

$$
\frac{\partial u_{m}}{\partial t}=U \frac{\partial u_{m}}{\partial x}+d_{m} \frac{\partial^{2} u_{m}}{\partial x^{2}}+e^{-\gamma \tau} u_{m}(x+U \tau, t-\tau)-\beta u_{m}^{2}
$$

and our derived equation as in (24) is studied when the species is close to extinction and the immature are not diffusing on homogeneous Dirichlet boundary conditions $u_{m}(0, t)=0, u_{m}(L, t)=0$ and the initial condition $u_{m}(x, 0)=u_{m}^{0} \geq 0$ for $x \in[-\tau, 0]$. Also, we considered the case when juveniles are not subject to advection, so the term $u_{m}(x+U \tau, t-\tau)$ in (24) becomes $u_{m}(x, t-\tau)$. However, the adults will still be subject to advection. The conditions on the parameters for the final solution $u_{m}(x, t)$ for the linearized (25) that prevents extinction of the species under the defect of advection for the reaction-diffusion are founds, where the conclusion can be summarized as follows:

- The species will become extinct if the advection speed if large, mature diffusion is either very small or very large, length of domain is small, juvenile mortality is large and length of juvenile is large.

- The species will survive if advection speed is small, domain is too large and birth rate is too large. 


\section{REFERENCES}

[1] Britton, N., "Reaction-Diffusion Equations and Their Applications to Biology," Academic Press, 1986.

[2] Lewis, M., Petrovskii, S., Potts, J., "Reaction-Diffusion Models: Single Species," The Mathematics Behind Biological Invasions, pp. 69-105, 2016.

[3] Allen, L., "Persistence and extinction in single-species reaction-diffusion models," Bulletin of Mathematical Bioloy, vol. 45, pp. 209-227, 1983.

[4] Aiello, W. G. and Freedman, H. I., "A time-delay model of single species growth with stage structure," Math. Biosci., vol. 101, pp. 139-153, 1990.

[5] Fisher, R. A., "The wave of advance of advantageous genes," Annals of Eugenics, vol. 7, pp. 355-369, 1937.

[6] Kolmogorov, A. Petrovskii, I. and Piscounov, N., "A study of the diffusion equation with increase in the amount of substance, and its application to a biological problem," In V. M. Tikhomirov, editor, Selected Works of A. N. Kolmogorov I, pages 248C270. Kluwer 1991. Translated by V. M. Volosov from Bull. Moscow Univ., Math. Mech. 1, pp. 1-25, 1937.

[7] Fisher R. A., "The genetical theory of natural selection," Oxford University Press, USA, New Ed edition 2000, variorum edition 1999, 1930.

[8] Gurney, W. S. C. and Nisbet, R. M., "Fluctuation periodicity, generation separation, and the expression of Larval competition," J. Theoretical Popul. Biol., vol. 28, no. 2, pp. 150-180, 1985.

[9] Ablowitz, M. J. and Zeppetella, A., "Explicit solutions of Fisher's equation for a special wave speed," Bull. Math. Biol., vol. 41, pp. 835-840, 1979.

[10] Al-Omari, J. F. M. and Gourley S. A., "A nonlocal reaction-diffusion model for a single species with stage structure and distributed maturation delay," Euro. J. Appl. Math., vol. 16, no. 1, pp. 37-51, 2005.

[11] Al-Omari, J., Gourley, S., "Dynamics of a stage-structured population model incorporating a state-dependent maturation delay," Nonlinear Anal. Real World Appl., vol. 6, no. 1, pp. 13-33, 2005.

[12] Aiello, W. G., Freedman, H. I. and Wu, J., "Analysis of a model representing stage-structured population growth with state-dependent time delay,” SIAM J. Appl. Math., vol. 52, pp. 855-869, 1992.

[13] Gourley, S. A., Jennings, R. and Liu, R., "An Advection and Age-Structured Approach to Modeling Bird Migration and Indirect Transmission of Avian Influenza," SIAM J. Appl. Math., vol. 75, no. 4, pp. 1620-1647, 2005.

[14] Sarfaraz W, Madzvamuse A., "Classification of parameter spaces for a reaction diffusion model onstationary domains," Chaos Solitons Fractals, vol. 103, pp. 33-51, 2007.

[15] Chunwei W., "A stage-structured delayed reaction-diffusion model for A stage-structured delayed reactiondiffusion model for competition between two species," Electronic Theses and Dissertations, University of Louisville, 2013.

[16] Rattanakul, C., Lenbury, Y. and Suksamran, J., "Analysis of advection-diffusion-reaction model for fish population movement with impulsive tagging: stability and traveling wave solution," Advances in Difference Equations, vol. 2019, 2019.

[17] Venkataraman, C., "Reaction-diffusion system on evolving dmains with applications to the theory of biological pattern formation," Ph.D. thesis, University of Sussex, 2011.

[18] Boonrangsiman, S., Bunwon, K. and Elvin, J., "A bifurcation path to chaos in a time-delay fisheries predator-prey model with prey consumption by immature and mature predators," Math. Computers Simul., vol. 124, pp. 16-29, 2016.

[19] Zhang, L. and Zhao, H. Y., "Traveling wave solutions in a stage-structured delayed reaction-diffusion model with advection," J. Math. Modelling Anal., vol. 20, no. 2, pp. 168-187, 2015.

[20] Cangiani, A., Georgoulis, E., Morozov, A. and Sutton O., "Revealing new dynamical patterns in a reactiondiffusion model with cyclic competition via a novel computational framework," Proc.R.Soc.A474, 2018

[21] Murray, J. D., "Mathematical Biology," 2nd ed., Springer, Berlin, 1993.

[22] Murray, J. D., "Mathematical Biology," I. An introduction. 3rd ed. Inter, disciplinary Applied Mathematics, Springer, New York, 2002.

[23] Murray, J. D. , "Mathematical biology," Spatial models and biomedical applications. Springer, New York, 2013.

[24] Metz, J. A. J and Diekmann, O., "The Dynamics of Physiologically Structured Populations," Springer-Verlag, New York, 1986.

[25] McNair, J., N., and Goulden, C. E., "The dynamics of age-structured populations with a gestation period: Densityindependent growth and egg ration methods for estimating the birth rate," Theoret. Population Bio., vol. 39, pp. 1-29, 1991. 